\title{
Surgical Treatment of a Young Athlete with Displaced Avulsion Fracture of the Anterior Superior Iliac Spine (ASIS)
}

\author{
Alexander E Poor , Alec Warren, Nicole Cannon and William C Meyers \\ Vincera Institute, Philadelphia, USA
}

*Corresponding author: Alexander E Poor, MD, Vincera Institute, 1200 Constitution Ave, Philadelphia, PA, 19112, USA, Tel: 1-267-592-3200, ORCID iD: 0000-0002-8275-9381

\begin{abstract}
Introduction: Avulsion fractures of the anterior superior iliac spine are uncommon without established treatment guidelines. Deciding between nonoperative versus surgical management depends on the grade of displacement, skeletal maturity, type of sport/activity, and competitive/ career requirements.

Methods: We present a young athlete who sustained an ASIS avulsion fracture while playing baseball that was treated with open surgical reduction and fixation using absorbable sutures with an emphasis on mobilizing the TFL to reapproximate the avulsed segment without tension.

Results: The patient was started on a progressive physical therapy protocol that did not involve any weight-bearing or range of motion restrictions. He was jogging in the third postoperative week and returned to full-play in the $6^{\text {th }}$ week. As of 12 months postop, there were no complications and he is playing at his pre-injury level of performance without pain.

Conclusion: Operative treatment of avulsion fracture of the ASIS without bone anchors or screws highlights the importance of soft tissue mobilization and reduction of tension. Additionally, the relatively rapid return to activities and lack of immobilization minimizes deconditioning, which might be an advantage over non-operative management. Athletes with ASIS avulsion fractures repaired with this technique can expect to return to play within 6 weeks.
\end{abstract}

\section{Keywords}

Tensor fascia latae (TFL), Anterior superior iliac spine (ASIS), Avulsion fracture, Core muscle injury

\section{List of Abbreviations}

ASIS: Anterior Superior lliac Spine; TFL: Tensor Fasciae Latae; MRI: Magnetic Resonance Imaging

\section{Introduction}

Avulsion fractures of the anterior superior iliac spine are uncommon and there are no established treatment guidelines. The decision between nonoperative versus surgical management depends on the grade of displacement, skeletal maturity, type of sport/activity, and competitive/career requirements.

\section{High-level athletes}

The Anterior Superior lliac Spine (ASIS) serves as the origin of the sartorius muscle and of parts of the Tensor Fasciae Latae (TFL). Avulsion injuries of the ASIS constitute $1.4 \%$ of injuries of the hip and pelvis and $19 \%$ of avulsion injuries of the pelvis [1,2]. A sudden forceful strain on the sartorius or the tensor fasciae latae during the starting phase of running or jumping is the most common mechanism of accident. Apophysis ossification of the ASIS usually occurs between the age of 21 and 25. Prior to the ossification, the epiphyseal plate is weaker than the myotendonous junction or pelvic bone, making avulsions more likely in younger athletes [3].

We present the case of a young athlete who sustained an ASIS avulsion fractures while playing baseball that was treated with open surgical reduction and fixation using absorbable sutures with an emphasis on mobilizing the tensor fascia latae to reapproximate the avulsed segment without tension.

\section{Case Report}

The patient is a 16 -year-old, Risser stage 2 , high-lev-

Citation: Poor AE, Warren A, Cannon N, Meyers WC (2019) Surgical Treatment of a Young Athlete with Displaced Avulsion Fracture of the Anterior Superior Iliac Spine (ASIS). Int J Sports Exerc Med 5:127. doi.org/10.23937/2469-5718/1510127

Accepted: April 27, 2019; Published: April 29, 2019

Copyright: (C) 2019 Poor AE, et al. This is an open-access article distributed under the terms of the Creative Commons Attribution License, which permits unrestricted use, distribution, and reproduction in any medium, provided the original author and source are credited. 

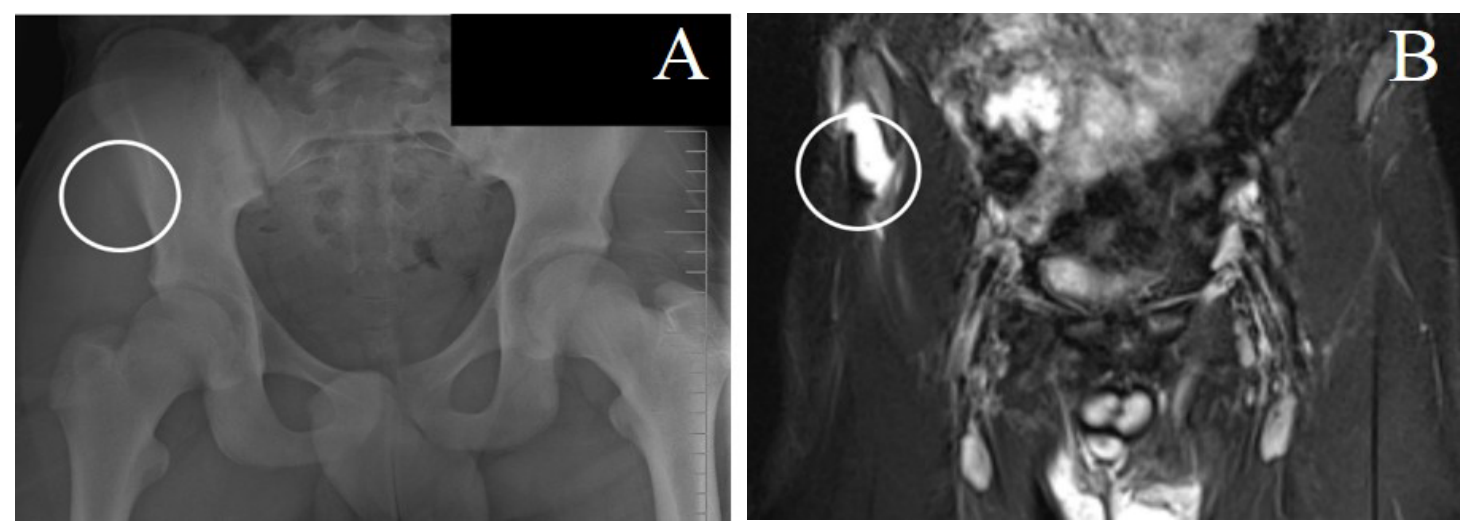

Figure 1: (A) X-ray; (B) MRI (STIR) demonstrating the avulsed ASIS. The bone fragment is displaced inferolaterally (white circle).
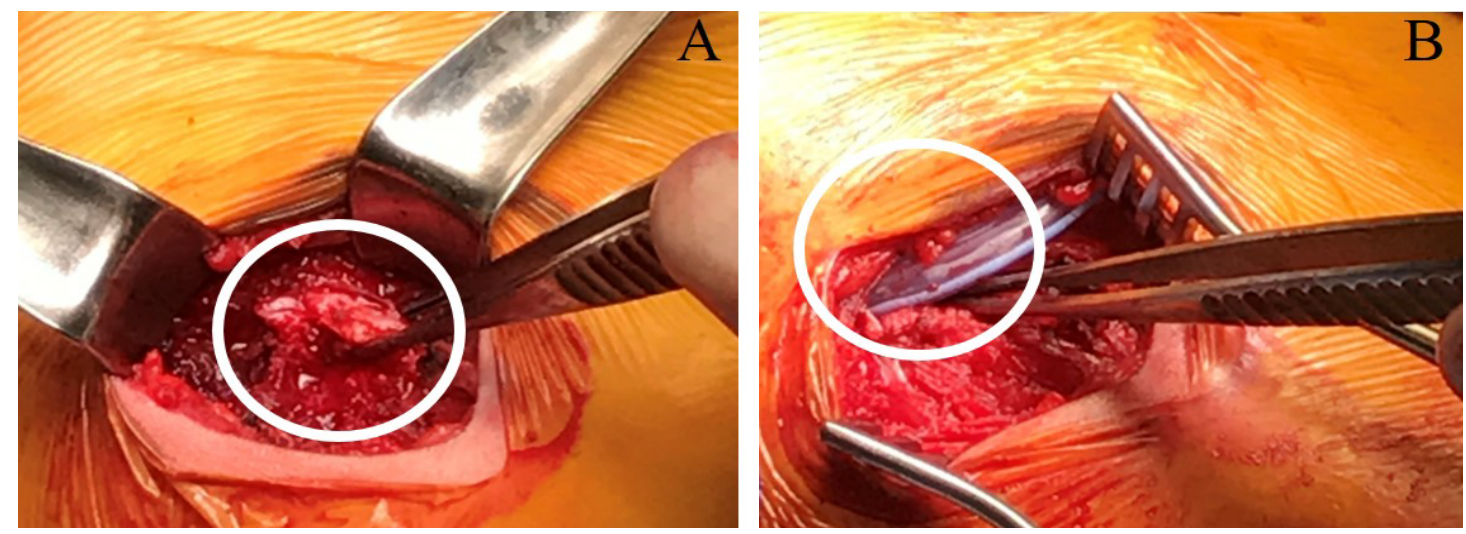

Figure 2: Intraoperative photos (A) The avulsed bone fragment with attached TFL and sartorius (white circle) is held in the forceps; (B) The forceps point to the location of the bone chip (white circle) after it has been reapproximated. A blake drain is superficial to the repair.

el high school baseball player who felt a pop and sudden, sharp pain at the right ASIS while simultaneously running the base path and shifting his hips to his right to avoid a tag [4]. Upon examination two days later, he had profound weakness and pain with right hip flexion. Pelvic $x$-ray and magnetic resonance imaging (Figure $1 \mathrm{~A}$ and Figure 1B) demonstrate a $2.5 \mathrm{~cm}$ avulsion of the right TFL with a $2.2 \times 0.8 \mathrm{~cm}$ chip of bone displaced inferolaterally from the ASIS and an intervening hematoma.

\section{Surgical technique}

The procedure was performed under general anaesthesia with Laryngeal Mask Airway (LMA) intubation. The patient was not given paralytics so that resting tone would allow better approximation of the tension on the muscles. The patient was placed in supine position and the anterolateral pelvis and thigh was prepped and draped. A vertical $3 \mathrm{~cm}$ skin incision was made below and just lateral to the ASIS. The fascia was divided longitudinally while taking care to protect the lateral femoral cutaneous nerve. This allowed entry into the hematoma cavity and palpation of the bony fragment distally. The origins of the sartorius muscle and the tensor fascia lata remained attached to the flake (Figure 2A). Following a combination of blunt and sharp dissection within the fascial compartment of the TFL and along the TFL and sartorius fascia, the bone fragment could be reapproximated to the avulsion site (Figure 2B). Absorbable sutures (0-PDS) were then placed between the myontendonous origins of the TFL and sartorious and the surrounding periosteum and soft tissue (external oblique, intact iliotibial band fibers, and gluteus maximus). A blake drain was placed on top of the repair.

\section{Postoperative course}

The patient was started on a progressive physical therapy protocol that did not involve any weightbearing or range of motion restrictions. The drain was removed on postoperative day 6 . He was jogging in the third postoperative week and returned to full play in the $6^{\text {th }}$ week. As of nine months postop, there were no complications and he is playing at his pre-injury level of performance without pain.

\section{Discussion}

There are no established treatment guidelines for avulsion fractures of the ASIS in the literature. The two most-commonly recommended treatment options are non-operative management and open reduction with screw or anchor fixation. Non-operative treatment can involve immobilization with the hip and knee in flexion 
to off-load the area of injury $[5,6]$. This combined with NSAIDs and subsequent gradual mobilization is a commonly-reported approach for non-displaced or minimally displaced fractures. Open reduction and screw fixation has been recommended in cases involving greater displacement of the bone fragment. Surgical treatment has been associated with a shorter rehabilitation period allowing an earlier return to athletic activities [7]. In a comparison of 23 adolescent patients with avulsion fractures of the ASIS of whom 10 were treated conservatively and 13 operatively, the final outcome after 1 -year-did not show any differences between the two treatment groups as assessed by the visual analog scale and range of motion [1].

The history of trauma, clinical examination, and standard radiographs can be sufficient for a correct diagnosis. The avulsed fragment usually is displaced distally and laterally [8]. In addition to standard radiography, Magnetic Resonance Imaging (MRI) is useful in cases with inconclusive X-ray findings [9]. Avulsed ASIS usually have an associated hematoma and severe cases with extensive callous formation have been mistaken for bone tumors or infectious processes [10]. Severely displaced fragments may also lead to compression of the lateral cutaneous nerve causing meralgia paraesthetica $[11,12]$. MRI is also helpful in defining the extent of the soft tissue injury and the structures involved.

Operative treatment of avulsion fracture of the ASIS without bone anchors or screws highlights the importance of soft tissue mobilization and reduction of tension on these repairs. Applying mobilization techniques, we have developed in treating adductor avulsion injuries reduces tension on the repair and obviates the need for bone fixation. Additionally, like the surgical approach described by Wilinger, et al. [3], this surgical approach does not violate the chondral growth zone of the pelvic apophysis and allows the injury to heal with less of a risk of growth disturbances as compared to screw fixation through the bone fragment [12]. The relatively rapid return to activities and lack of immobilization minimizes deconditioning, which might be an advantage over non-operative management. Athletes with ASIS avulsion fractures repaired with this technique can expect to return to play within 6 weeks.

\section{References}

1. Kautzner J, Trc T, Havlas V (2014) Comparison of conservative against surgical treatment of anterior-superior iliac spine avulsion fractures in children and adolescents. Int Orthop 38: 1495-1498.

2. Rossi F, Dragoni S (2001) Acute avulsion fractures of the pelvis in adolescent competitive athletes: prevalence, location and sports distribution of 203 cases collected. Skeletal Radiol 30: 127-131.

3. Willinger L, Schanda JE, Lorenz S, Imhoff AB, Buchmann $S$ (2017) Surgical treatment of two adolescent athletes with dislocated avulsion fracture of the anterior superior iliac spine (ASIS). Arch Orthop Trauma Surg 137: 173-177.

4. Risser JC (1958) The iliac apophysis; an invaluable sign in the management of scoliosis. Clin Orthop 11: 111-119.

5. Kujala UM, Orava S (1993) Ischial apophysis injuries in athletes. Sports Med 16: 290-294.

6. Poitinger H, Munk P, Poeschl GP (2003) Avulsion fracture of the anterior superior iliac spine following apophysitis. $\mathrm{Br}$ J Sports Med 37: 361-362

7. Cimerman M, Smrkolj V, Veselko M (1995) Avulsion of the anterior superior iliac spine in two adolescent sisters: operative versus conservative treatment. Unfallchirurg 98: 530-531.

8. Hansson G (1970) Bilateral avulsion fracture of the anterior superior iliac spine. Report of a case. Acta Chir Scand 136: 85-86.

9. Naylor JA, Goffar SL, Chugg J (2013) Avulsion fracture of the anterior superior iliac spine. J Orthop Sports Phys Ther 43: 195

10. Dhinsa BS, Jalgaonkar A, Mann B, Butt S, Pollock R (2011) Avulsion fracture of the anterior superior iliac spine: misdiagnosis of a bone tumour. J Orthop Traumatol 12: 173-176.

11. Hayashi S, Nishiyama T, Fujishiro T, Kanzaki N, Kurosaka M (2011) Avulsion-fracture of the anterior superior iliac spine with meralgia paresthetica: a case report. J Orthop Surg (Hong Kong) 19: 384-385.

12. Hsu CY, Wu CM, Lin SW, Cheng KL (2014) Anterior superior iliac spine avulsion fracture presenting as meralgia paraesthetica in an adolescent sprinter. J Rehabil Med 46: 188-190. 